\title{
Detection of Klebsiella pneumoniae cfDNA in pleural fluid and its clinical value
}

\author{
Min Ren ${ }^{1}, \mathrm{Li} \mathrm{Li}{ }^{1}$, Mingliang Chu ${ }^{1}$, Lin $\mathrm{Liu}^{2}$ \\ ${ }^{1}$ Department of Clinical Laboratory, First Affiliated Hospital of Guizhou University of Traditional Chinese Medicine, Guiyang, China; ${ }^{2}$ Department \\ of Respiratory and Critical Care Medicine, Guizhou Provincial People's Hospital, Guiyang, China \\ Contributions: (I) Conception and design: M Chu; (II) Administrative support: M Ren, L Liu; (III) Provision of study materials or patients: M Ren, L \\ Li; (IV) Collection and assembly of data: M Ren, L Li; (V) Data analysis and interpretation: L Li; (VI) Manuscript writing: All authors; (VII) Final \\ approval of manuscript: All authors. \\ Correspondence to: Mingliang Chu. Department of Clinical Laboratory, First Affiliated Hospital of Guizhou University of Traditional Chinese \\ Medicine, 71 Baoshanbei Road, Guiyang, China. Email: chumingliang760@gzy.edu.cn.
}

Background: Klebsiella pneumoniae (KP) is an important opportunistic pathogen that can easily cause pneumonia and pleural effusion when body resistance is reduced. However, the positive rate of KP detected from clinical pleural effusion by traditional methods, including bacterial culture, is meager. Therefore, new detection methods are urgently needed to improve the positive detection rate of KP and other bacteria in pleural effusion.

Methods: Simulated pleural fluid of KP infection was first set up. Then circulating cell-free DNA (cfDNA) was extracted from cultured hydrothorax and detected by fluorescence polymerase chain reaction (PCR) to verify KP cfDNA in the pleural fluid. The specificity, sensitivity, and repeatability of this method are verified by detecting the cfDNAs in pleural effusion, samples of malignant pleural effusion, tuberculous pleural effusion, and other common microbial infections. Finally, this method was compared with three traditional methods, pleural effusion, precipitation DNA, sputum culture, and pleural effusion culture to explore the clinical diagnostic value of this method.

Results: KP cfDNA was positive by fluorescence PCR from the simulated KP infected pleural effusion, which confirmed KP cfDNA in pleural effusion. KP cfDNA was positive by fluorescence PCR from the pleural effusion of KP infected patients, while with the same detection method, KP cfDNA in clinical carcinomatous hydrothorax, tuberculosis hydrothorax, and other standard microbial infection samples was negative, which confirmed the method had high specificity, high sensitivity, and reproducibility. Compared with the three traditional methods, this method has a higher positive rate. Compared with the gold standard, sputum bacterial culture, the sensitivity, specificity, positive predictive value, and negative predictive value of this method were $91.67 \%, 95.45 \%, 91.7 \%$, and $95.5 \%$, respectively.

Conclusions: The detection of cfDNA by fluorescence PCR is possible. Moreover, the positive rate of this method in clinical pleural effusions is high.

Keywords: Klebsiella pneumoniae (KP); cell-free DNA (cfDNA); parapneumonic effusion (PE)

Submitted Jul 16, 2020. Accepted for publication Sep 04, 2020.

doi: 10.21037/apm-20-1574

View this article at: http://dx.doi.org/10.21037/apm-20-1574 


\section{Introduction}

Klebsiella pneumoniae (KP) is a gram-negative, rod-shape organism and encountered as a saprophyte in humans (1). Infections of KP are rare in healthy people, but are more likely in immunocompromised patients. A number of pathological conditions are associated with this bacterial infection such as pneumonia, bacteremia, septicemia, endocarditis, meningitis, and cellulitis (2). In the past, KP was considered an important causative agent of communityacquired infections. But from early 1970s, it began to become a major cause of hospital-acquired infections, including urinary tract infection, bloodstream infection, liver abscesses and pneumonia (it is a frequent cause of ventilator-associated pneumonia) (3). Though $\mathrm{KP}$ accounts for a small percentage of pneumonia cases, the case fatality rates are high (4). Studies also showed that KP was a dominant pathogen in complicated parapneumonic effusion (PE) (5). $\mathrm{PE}$ is a common complication of pneumonia (6-8). It was found about $40 \%$ of hospitalized pneumonia patients could be complicated with PE, which led to the poor treatment effect of pneumonia and increased mortality (9-13). Early choice of sensitive antibiotics is the primary method for the treatment of PE. The ideal method is to select the corresponding antibiotics according to the results of bacterial culture and in vitro drug sensitivity tests. However, the actual situation is that the positive detection rate of bacteria in clinical pleural effusion is meager, which may be related to antibiotic treatment and other factors (14). Sputum culture is the primary method for bacterial examination. However, KP is a conditional pathogen and exists typically in the upper respiratory tract of the human body. Oral and pharyngeal host bacteria often contaminate sputum culture, and the culture results cannot truly be the pathogens of pulmonary infection (15). Therefore, new detection methods are urgently needed to improve the positive detection rate of $\mathrm{KP}$ and other bacteria in pleural effusion. Cell-free DNA (cfDNA) is a type of extracellular DNA, also known as cfDNA or circulating DNA. cfDNA can be detected in pleural effusion, ascites, urine, prostatic fluid, synovial fluid, cerebrospinal fluid, and blood $(16,17)$. The study on free DNA discovered mature blood of tumor patients, while cfDNA can be used as an index for early detection, diagnosis, and later targeted drug use (17-21). In recent years, cfDNA has been found in the body fluids of patients infected with some pathogens, including Plasmodium, parasites, and Mycobacterium tuberculosis (22-26). Detection of free DNA $(25,27)$ of pathogens in body fluids of patients infected with Plasmodium and Schistosoma by PCR showed advantages in disease diagnosis and monitoring $(25,27)$. The detection of cfDNA of Mycobacterium tuberculosis in pleural effusion by fluorescence PCR can significantly improve the positive detection rate (22). However, up to now, there is no report on the detection of the free DNA of KP by PCR. Therefore, in this study, we looked for to set up the detection system of the free DNA of KP and explore its value in clinical application. We present the following article in accordance with the STARD reporting checklist (available at http://dx.doi.org/10.21037/apm-20-1574).

\section{Methods}

\section{Data collection}

According to the inclusion criteria of $\mathrm{PE}$, after lung tumor, pulmonary tuberculosis, pulmonary vasculitis, pulmonary embolism, atelectasis, noninfectious pulmonary interstitial disease, pulmonary edema, pulmonary eosinophilic infiltration, and other diseases were excluded, the clinical PE (residual hydrothorax specimens after routine medical examination) was collected from the First Affiliated Hospital of Guizhou University of Traditional Chinese Medicine from August 2019 to November 2019 and preserved at $-20{ }^{\circ} \mathrm{C}$. The study was conducted in accordance with the Declaration of Helsinki (as revised in 2013). The Ethics Committee approved the study of First Affiliated Hospital of Guizhou University of Traditional Chinese Medicine (K2020-001). Informed consent was waived according to the Office for Protection from Research Risk (OPRR) Guidelines, the second wavier condition of 45 CFR 46.116 section D: (I) the research involves no more than minimal risk to the subjects; (II) the waiver or alteration will not adversely affect the rights and welfare of the subjects.

After reviewing the clinical data, according to the research needs, we selected 13 cases of pleural effusion samples with positive sputum culture for KP (including 4 cases of pleural effusion culture that was also positive) and 22 cases of pleural effusion samples with negative sputum culture for KP and simultaneously reviewed the pleural fluid culture results. Also, pleural effusion from 3-10 patients with clinically confirmed malignant pleural effusion, tuberculous pleural effusion, and Staphylococcus aureus, Pseudomonas aeruginosa, Staphylococcus baemolyticus, Escherichia coli, Staphylococcus epidermidis, Enterococcus faecalis, Acinetobacter baumannii and Candida Albicans infected pleural effusion that was collected (residual hydrothorax specimens after routine medical examination). 


\section{Bacterial identification}

The sputum sample and pleural effusion were inoculated into the special culture bottle for culture. If automatic blood cultivator detected any bacterial colony, transferred them to a blood plate and MacConkey plate and picked out suspected colonies to identify them with automatic bacterial identification and analysis system.

\section{cfDNA extraction and PCR detection of simulated KP pleural effusion samples}

Three clear pleural effusions were randomly selected, $10 \mathrm{~mL}$ each. Five $\mathrm{mL}$ were inoculated with $\mathrm{KP}$ isolated and identified in our laboratory. The other $5 \mathrm{~mL}$ was used as a negative control. For all the pleural effusion samples, shaking culture was performed overnight at $37^{\circ} \mathrm{C}$ for about $16 \mathrm{~h}$. On the next day, the pleural effusion samples were centrifuged for 5 minutes at $12,000 \mathrm{rpm}$. The supernatant was then filtered with a disposable $0.22 \mu \mathrm{m}$ syringe filter. cfDNA in the supernatant was extracted according to the instructions of Circulate Nucleic Acids Kit (Amoy Diagnostics Co., LTD, Xiamen, China). The centrifuged precipitate was used to extract the total genomic DNA by the cell genomic DNA extraction Kit (Beijing Solarbio Science \& Technology Co., Ltd., Beijing, China).

\section{cfDNA extraction and PCR detection of clinical KP pleural effusion samples}

Five $\mathrm{mL}$ pleural effusion of patients with clinically confirmed PE stored at $-20^{\circ} \mathrm{C}$ in the early stage was centrifuged for 5 minutes at 12,000 rpm, and the supernatant was filtered with a disposable $0.22 \mu \mathrm{m}$ syringe filter. cfDNA in the supernatant was extracted and then centrifuged precipitate was used to extract the total genomic DNA.

\section{Specificity test}

cfDNA templates were prepared from the pleural effusions infected by Staphylococcus aureus, Mycobacterium tuberculosis, Staphylococcus haemolyticus, Staphylococcus epidermidis, Enterococcus faecalis, Acinetobacter baumannii, Pseudomonas aeruginosa, Escherichia coli, and Candida albicans, and common clinical cancerous pleural effusions, respectively, which were diagnosed by our laboratory earlier. The specificity was verified by KP fluorescence PCR.

\section{Sensitivity test}

The cfDNA of the simulated KP pleural effusion samples was continuously diluted by 10 times, $4 \mu \mathrm{L}$ was taken from each diluted sample for KP gene PCR amplification, and the minimum detection limit was determined.

\section{Repeatability test}

Three PCR positive cfDNA samples with high, medium, and low values were randomly selected for five times of repeated detection, and the coefficient of variation $(\mathrm{CV})$ value was calculated.

\section{PCR reaction conditions and interpretation criteria}

PCR was performed on LightCycler ${ }^{\circledR} 480$ II instrument (Roche Diagnostics, Rotkreuz, Switzerland). Response procedure according to instructions of KP fluorescence PCR assay kit (Shanghai ZJ Bio-Tech Co., Ltd., Shanghai, China): $37^{\circ} \mathrm{C}, 2 \mathrm{~min} ; 94^{\circ} \mathrm{C}, 2 \mathrm{~min}$; then $93{ }^{\circ} \mathrm{C}, 15 \mathrm{sec}$; $60{ }^{\circ} \mathrm{C}, 60 \mathrm{sec}$, total 40 cycles. The data were analyzed after amplification. Positive criteria: the curve showed or was close to "S," and the Ct values of both VIC and FAM signals were not more than 38 .

\section{Statistical methods}

SPASS 17.0 software was used for statistical analysis. $T$-test or chi-square test was used for comparison between groups, and the test level was $\alpha=0.05$.

\section{Results}

\section{Extraction and PCR validation of the cfDNA and precipitation from simulated KP pleural effusion}

KP grew well after overnight culture in the cancerous pleural effusion, which became turbid, while the pleural effusion in the negative control group was still apparent. Fluorescence quantitative PCR showed that the total DNA of the cfDNA and the precipitation from the three simulated pleural effusions inoculated with KP were all positive, while those of three negative control samples were all negative. 
Table 1 Comparison of detection results of KP by different methods

\begin{tabular}{|c|c|c|c|c|c|c|}
\hline \multirow{2}{*}{ Sputum culture } & \multicolumn{2}{|c|}{ Supernatant pleural effusion cfDNA } & \multicolumn{2}{|c|}{ Pleural effusion precipitation DNA } & \multicolumn{2}{|c|}{ Pleural effusion culture } \\
\hline & + & - & + & - & + & - \\
\hline+ & 11 & 1 & 2 & 10 & 4 & 8 \\
\hline
\end{tabular}

+, positive; -, negative. KP, Klebsiella pneumoniae; cfDNA, cell-free DNA.

\section{cfDNA extraction and PCR verification of clinical $K P$ pleural effusion samples}

In 4 cases, the inflammatory pleural effusion samples were confirmed to be KP positive in the later stage of pleural fluid culture. The cfDNA and precipitated DNA in the early preserved pleural effusion samples were detected by quantitative fluorescence PCR. The PCR results showed that the cfDNA was positive in all the four samples, while only 3 of the precipitated DNA were positive.

\section{Specificity, sensitivity, and reproducibility test}

This study showed that the detection system was specific to KP. The detection results were negative for any clinical common malignant pleural effusion, tuberculous pleural effusion, and the pleural effusions caused by Staphylococcus aureus, Mycobacterium tuberculosis, Staphylococcus haemolyticus, Staphylococcus epidermidis, Enterococcus faecalis Acinetobacter baumannii, Pseudomonas aeruginosa, Escherichia coli, and Candida albicans infection. The results showed that the detection limit was $0.001 \mathrm{ng}$ for the qualitative amplification of $\mathrm{KP}$ cfDNA in pleural effusion. The gradient dilution concentration of the cfDNA was negatively correlated with the corresponding PCR Ct value ( $r=0.93)$, which showed that the method had a high sensitivity. In the experiment, different cfDNA samples with PCR Ct values of 24, 28 and 32 respectively were selected for five repeated detections, and the $\mathrm{CV}$ values were $3.7 \%, 4.3 \%$ and $4.9 \%$ respectively, which met the industry standard deviation requirements of National Center of Clinical Laboratories.

\section{Comparison of the results from different detection methods for clinical KP samples}

We selected 13 cases of pleural effusion samples with positive sputum culture for KP (including 4 cases of pleural effusion culture was also positive) and 22 cases of pleural effusion samples with negative sputum culture for KP according to the research needs. Compared with the results of the sputum culture method (Table 1), the positive rates were the highest $(11 / 12)$ in the supernatant pleural effusion cfDNA method, the lowest in the pleural effusion precipitation DNA method $(2 / 12)$ and much lower in the pleural effusion culture method (3/12). At the same time, the cfDNA was also positive in 4 cases of pleural effusion culture. Also, in one case, the cfDNA was positive in the sputum culture method, but negative in the supernatant pleural effusion method was negative; in another case, the cfDNA was negative in the sputum culture method, but positive in the supernatant pleural fluid method. On the whole, the positive detection rate of the supernatant pleural effusion cfDNA method by PCR was much higher than that of the pleural effusion precipitation DNA method $(\mathrm{P}<0.05)$ and the pleural effusion culture method $(\mathrm{P}<0.05)$. There was no significant difference between the supernatant pleural effusion cfDNA method by PCR and the sputum culture method $(\mathrm{P}>0.05)$. Compared with the results of the sputum culture, the sensitivity, specificity, positive predictive value, and negative predictive value of quantitative fluorescence PCR were 91.67\%, 95.45\%, 91.7\%, and 95.5\%, respectively.

\section{Discussion}

$\mathrm{PE}$ is a common complication of pneumonia. However, it was found the positive rate of bacteria in which pleural effusion was meager $(14,28,29)$ in the clinic, which may be related to using antibiotics before submission. Therefore, it is necessary to improve the method to increase the positive detection rate of bacteria in pleural effusion. cfDNA is disintegrating DNA from cells and pathogens in the process of apoptosis or dying, which can be shed into body fluids, including blood, cerebrospinal fluid, urine, pleural, and ascites $(16-22,24)$. Therefore, in theory, the cfDNA of pathogens in pleural effusion is not affected even if antibiotics are used. In this study, KP cfDNA in pleural effusion was the first to be confirmed. Therefore, this experiment confirmed that KP cfDNA was indeed in pleural effusion and could be detected by PCR. To further confirm the establishment of this system, we specially selected $4 \mathrm{PE}$ samples with $\mathrm{KP}$ positive results in 
pleural effusion culture. PCR was used to detect the cfDNA, and the results were all positive. We also found that only 2 cases of DNA precipitation results were positive, showing that the sensitivity of the cfDNA detection results was higher than precipitated DNA results. Also, in clinical pleural effusion samples, the detection system only amplifies KP, with high specificity. It was confirmed KP cfDNA existed in pleural effusion, and the detection system had high specificity, sensitivity, and repeatability. However, the sample size was small, further large scale studies should be undertaken to validate these findings.

Some researchers believe that, theoretically, the pathogens of pulmonary infection are consistent with the pathogens in the pleural cavity, but the microenvironment of the lung tissue and the pleural cavity is not entirely the same. Therefore, the pathogens in PE may be different from pulmonary infection (15). Moreover, KP is a conditional pathogen, which typically exists in the upper respiratory tract of the human body. The bacteria also contaminate the clinical sputum culture colonized in the oropharynx, and the culture results cannot represent the pathogens of pulmonary infection (15). Therefore, clinical diagnosis and treatment should be on the results of pleural effusion detection. The results also confirmed the positive results of cfDNA detection in the pleural effusion of $\mathrm{KP}$ infection, were consistent with those of sputum culture and were much higher than those of pleural effusion culture and pleural effusion precipitation DNA detection. It was found that sputum culture was positive, but cfDNA was negative. We hypothesize that that specimen may be a contaminated specimen of the healthy upper respiratory tract. Sputum culture-negative but cfDNA positive specimens suggest to clinicians that the pathogens of pulmonary infection are not consistent with those in the pleural cavity.

In conclusion, the KP cfDNA detection system was set up in this study, and the feasibility and clinical application value of the system were confirmed.

\section{Acknowledgments}

Finding: This study was supported by the National Natural Science Foundation of China (Grant No. 81560003).

\section{Footnote}

Reporting Checklist: The authors have completed the STARD reporting checklist. Available at http://dx.doi.org/10.21037/ apm-20-1574

Data Sharing Statement: Available at http://dx.doi. org/10.21037/apm-20-1574

Conflicts of Interest: All authors have completed the ICMJE uniform disclosure form (available at http://dx.doi. org/10.21037/apm-20-1574). The authors have no conflicts of interest to declare.

Ethical Statement: The authors are accountable for all aspects of the work in ensuring that questions related to the accuracy or integrity of any part of the work are appropriately investigated and resolved. The study was conducted in accordance with the Declaration of Helsinki (as revised in 2013). The Ethics Committee approved the study of First Affiliated Hospital of Guizhou University of Traditional Chinese Medicine (K2020-001). Informed consent was waived according to the Office for Protection from Research Risk (OPRR) Guidelines, the second wavier condition of 45 CFR 46.116 section D: (I) the research involves no more than minimal risk to the subjects; (II) the waiver or alteration will not adversely affect the rights and welfare of the subjects.

Open Access Statement: This is an Open Access article distributed in accordance with the Creative Commons Attribution-NonCommercial-NoDerivs 4.0 International License (CC BY-NC-ND 4.0), which permits the noncommercial replication and distribution of the article with the strict proviso that no changes or edits are made and the original work is properly cited (including links to both the formal publication through the relevant DOI and the license). See: https://creativecommons.org/licenses/by-nc-nd/4.0/.

\section{References}

1. Karruli A, Andini R, Corcione A, et al. Prevention and control of intensive care unit-acquired carbapenemresistant Klebsiella pneumoniae: need for a multimodal approach. Ann Transl Med 2019;7:S325.

2. Tumbarello M, Trecarichi EM, De Rosa FG, et al. Infections caused by KPC-producing Klebsiella pneumoniae: differences in therapy and mortality in a multicentre study. J Antimicrob Chemother 2015;70:2133-43.

3. Tzouvelekis LS, Markogiannakis A, Psichogiou M, et al. Carbapenemases in Klebsiella pneumoniae and 
other Enterobacteriaceae: an evolving crisis of global dimensions. Clin Microbiol Rev 2012;25:682-707.

4. Vading M, Nauclér P, Kalin M, et al. Invasive infection caused by Klebsiella pneumoniae is a disease affecting patients with high comorbidity and associated with high long-term mortality. PLoS One 2018;13:e0195258.

5. Lin YT, Chen TL, Siu LK, et al. Clinical and microbiological characteristics of community-acquired thoracic empyema or complicated parapneumonic effusion caused by Klebsiella pneumoniae in Taiwan. Eur J Clin Microbiol Infect Dis 2010;29:1003-10.

6. Hong JY, Park SY, Kim Y, et al. Calpain and spectrin breakdown products as potential biomarkers in tuberculous pleural effusion. J Thorac Dis 2018;10:2558-66.

7. Chalmers JD, Singanayagam A, Murray MP, et al. Risk factors for complicated parapneumonic effusion and empyema on presentation to hospital with communityacquired pneumonia. Thorax 2009;64:592-7.

8. Li D, Shen Y, Qin J, et al. Diagnostic performance of C-reactive protein for parapneumonic pleural effusion: a meta-analysis. Ann Transl Med 2019;7:1.

9. Huang Y, Jiao Y, Zhang J, et al. Microbial etiology and prognostic factors of ventilator-associated pneumonia: a multicenter retrospective study in Shanghai. Clin Infect Dis 2018;67:S146-S152.

10. Meyer Sauteur PM, Burkhard A, Moehrlen U, et al. Pleural tap-guided antimicrobial treatment for pneumonia with parapneumonic effusion or pleural empyema in children: a single-center cohort study. J Clin Med 2019;8:698.

11. Segerer FJ, Seeger K, Maier A, et al. Therapy of 645 children with parapneumonic effusion and empyema-A German nationwide surveillance study. Pediatr Pulmonol 2017;52:540-7.

12. Griffith D, Boal M, Rogers T. Evolution of practice in the management of parapneumonic effusion and empyema in children. J Pediatr Surg 2018;53:644-6.

13. Bengoechea JA, Sa Pessoa J. Klebsiella pneumoniae infection biology: living to counteract host defences. Fems Microbiol Rev 2019;43:123-44.

14. Sahn SA. Diagnosis and management of parapneumonic effusions and empyema. Clin Infect Dis 2007;45:1480-6.

15. Strange C, Sahn SA. The definitions and epidemiology of pleural space infection. Semin Respir Infect 1999;14:3-8.

16. Gorgannezhad L, Umer M, Islam MN, et al. Circulating tumor DNA and liquid biopsy: opportunities, challenges, and recent advances in detection technologies. Lab Chip 2018;18:1174-96.

17. Zeng H, He B, Yi C, et al. Liquid biopsies: DNA methylation analyses in circulating cell-free DNA. J Genet Genomics 2018;45:185-92.

18. Fece de la Cruz F, Corcoran RB. Methylation in cell-free DNA for early cancer detection. Ann Oncol 2018;29:1351-3.

19. Feng LX, Wang J, Yu Z, et al. Clinical significance of serum EGFR gene mutation and serum tumor markers in predicting tyrosine kinase inhibitor efficacy in lung adenocarcinoma. Clin Transl Oncol 2019;21:1005-13.

20. Panagopoulou M, Karaglani M, Balgkouranidou I, et al. Circulating cell-free DNA in breast cancer: size profiling, levels, and methylation patterns lead to prognostic and predictive classifiers. Oncogene 2019;38:3387-401.

21. Wu FT, Lu L, Xu W, et al. Circulating tumor DNA: clinical roles in diffuse large B cell lymphoma. Ann Hematol 2019;98:255-69.

22. Che N, Yang X, Liu Z, et al. Rapid Detection of Cell-Free Mycobacterium tuberculosis DNA in Tuberculous Pleural Effusion. J Clin Microbiol 2017;55:1526-32.

23. Weerakoon KG, McManus DP. Cell-Free DNA as a diagnostic tool for human parasitic infections. Trends Parasitol 2016;32:378-91.

24. Blauwkamp TA, Thair S, Rosen MJ, et al. Analytical and clinical validation of a microbial cell-free DNA sequencing test for infectious disease. Nat Microbiol 2019;4:663-74.

25. Weerakoon KG, Gordon CA, Williams GM, et al. Droplet digital PCR diagnosis of human schistosomiasis: parasite cell-Free DNA detection in diverse clinical samples. J Infect Dis 2017;216:1611-22.

26. Johansson N, Vondracek M, Backman-Johansson C, et al. The bacteriology in adult patients with pneumonia and parapneumonic effusions: increased yield with DNA sequencing method. Eur J Clin Microbiol Infect Dis 2019;38:297-304.

27. Gal S, Wainscoat JS. Detection and quantitation of circulating Plasmodium falciparum DNA by polymerase chain reaction. Methods Mol Biol 2006;336:155-62.

28. Franchetti L, Schumann D M, Tamm M, et al. Multiplex bacterial polymerase chain reaction in a cohort of patients with pleural effusion. BMC Infect Dis 2020;20:99.

29. Li C, Hou L, Sharma BY, et al. Developing a new intelligent system for the diagnosis of tuberculous pleural effusion. Comput Methods Programs Biomed 2018;153:211-25.

(English Language Editor: J. Chapnick)

Cite this article as: Ren M, Li L, Chu M, Liu L. Detection of Klebsiella pneumoniae cfDNA in pleural fluid and its clinical value. Ann Palliat Med 2020;9(5):3379-3384. doi: 10.21037/apm-20-1574 